\title{
Innovative technologies in the upgrading of existing r.c. framed structures
}

\author{
L. Anania \& G. D’Agata \\ Department of Civil Engineering and Architecture, \\ University of Catania, Italy
}

\begin{abstract}
Many r.c. structures have to be strengthened or retrofitted to resist higher design loads. This happens when there is a change of the use destination or when the structures are damaged because of an aggressive environment. Besides, nowadays, attention has moved to restoring, recovering and seismically retrofitting the r.c. structures The knowledge that r.c. is alone, not always, capable of reaching the structural seismic requirements has given the drive to combine it together with an innovative material such as CFRP. That is, the strengthening of the r.c. structural elements by using externally bonded CFRP, which seems to be an effective solution and also the most economical one, over a long period of time. But sometimes, the compressed zone of concrete fails because it is not capable of sustaining the new stress state produced by the increase of resistance of the structural element. In this paper we discuss an innovative technology, consisting of gluing a joist of polyurethane, at the internal face of the frame mesh and wrapped by CFRP layers. So an increasing of its flexural capacity and stiffness, while maintaining a manageable member weight, can be reached. Once strengthened, this will show a ductility capable of resisting the horizontal action by avoiding the introduction, either of the very heavy r.c. layers or the steel plates. We report the results of an experimental test compared with non-strengthened-frame ones. The hysteretical loops of the reinforced frame, derived from the tests, are sensibly wider, so this retrofitting technology seems to be very similar to the technology consisting of applying, in frame mesh, some hysteretical devices which permit an unloading of the seismic energy from the main structure. For this reason the same original frame was tested coupled with a particular device designed tested and patented by the same author. In this
\end{abstract}


way, a comparison between the technique based on the use of CFRP and the one based on the use of the hysteretical devices has been possible and discussed. Keywords: CFRP, r.c. rehabilitation, Composite materials, new technology.

\section{Introduction}

C-FRP was used for a long time in both naval and aeronautic constructions since carbon fibers are very light materials and have a very high resistance which is not comparable neither to the steel nor to r.c.. The CFRP materials offer a flexural shear and traction resistance hundreds of times greater than the traditional r.c., and many tests and a wide range of experimental investigations have shown the efficiency of their application to the reinforced concrete members (Galicia et al. [1], Pendhari et al. [2], Della Corte et al. [3]). In this work we try to give a new scientific contribution in terms of both the experimental and the analytical study in the use of carbon fibers applied to civil structures. To this aim, the investigation was carried out on a damaged and then repaired r.c. frame. The behavior of the tested structure was firstly examined both in the absence and in the presence of the CFRP. The innovative technology, proposed in the paper, consists of applying the CFRP (Anania et al. [4]) by means of the interposition of a thin element of polyurethane glued at the internal face of the frame mesh (as shown in figure 1). The polyurethane is deprived of any structural feature but it has the scope of centrifuging the CFRP layer so as to increase the flexural resistant required for the retrofitting. This technology was already tested by the same authors on a single r.c. beam, and it has given excellent results [1].

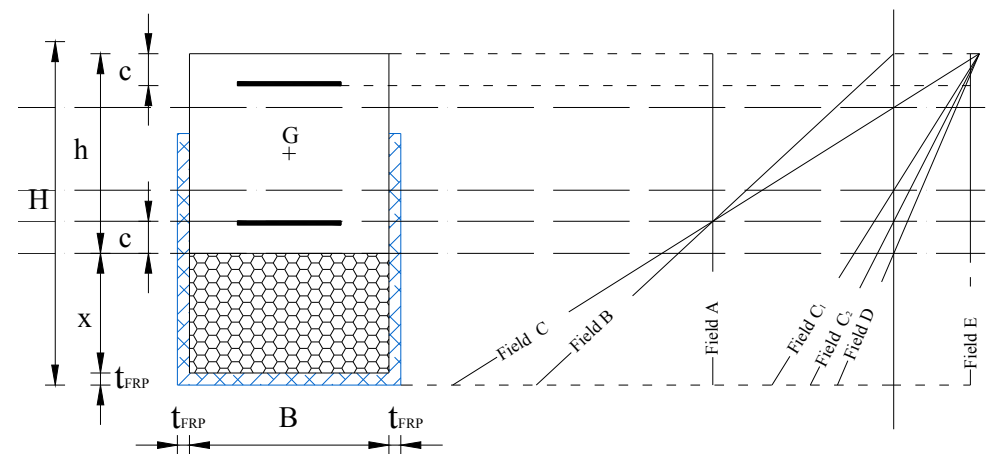

Figure 1: Limit fields for the strengthened composite section.

\section{The analytical approach}

The resistance domains were determined by referring to the "limit states" analysis in agreement with European and American rules Aci 440 applied to r.c. cross sections, but also considering the contribution of both the polyurethane and 
the CFRP material. The scope of this investigation is to prove the enhancement of the flexural resistance along the cross section due to the proposed technology. The basic hypothesis is the plane conservation of the cross sections.

A previous study (Anania and D'Agata [5]) were focused to determine a suitable polyurethane thickness capable of ensuring the plane section conservation once the limit yield was exceed. We found that a right thickness of the joist must be not greater than $0.28 \mathrm{H}$, where " $\mathrm{H}$ " represents the total height of the cross section.

Figures 2 and 3 show the enhancement obtained in terms of $\mathrm{M}-\mathrm{N}$ domain when the CFRP layer is increased.

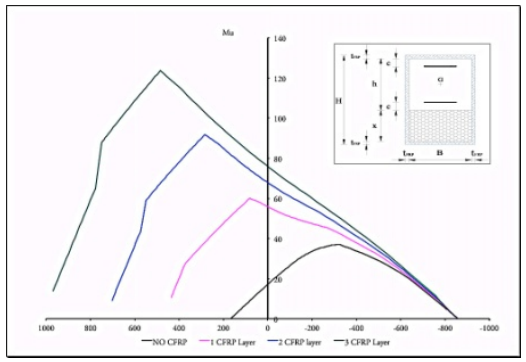

Figure 2: Bearing capacity of the column cross sections vs. no. C-FRP layers.

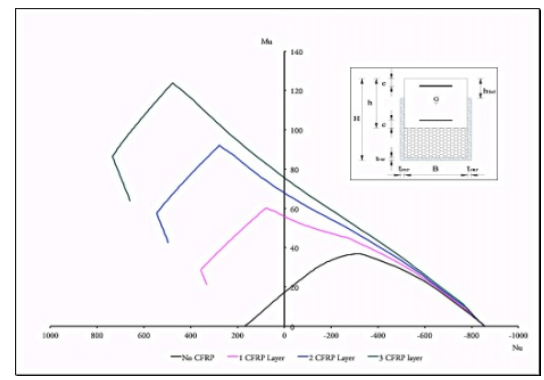

Figure 3: Bearing capacity of the beam cross sections vs. no. CFRP layers.

\section{Experimental validation of the proposed system}

The proposed technology was tested on an r.c. frame scaled 1:2 size. The force displacement curve obtained was compared with the one related to the virgin frame.

\subsection{Virgin frame}

At first, the test was carried out on an r.c. frame scaled 1:2 in size. The dimensions of each sample were $240 \times 170 \mathrm{~cm}$. The cross section of both the columns and the beam was $25 \times 25 \mathrm{~cm}^{2}$ reinforced by four steel bars B450C of $\phi 12 \mathrm{~mm}$ in diameter. The model, represented in figure 4, was subjected to load test up to the collapse firstly by means of monotonic loading and then, after its repairing, by cyclic load.

The load displacement curves are reported in figure 5 both for monotonic tests and for cyclic tests. The vertical load was constant at $100 \mathrm{kN}$, and it was applied by means of hydraulic jacks located on the top of each column. The horizontal load was applied by a two-directional jack at digital control. The collapse mechanisms was caused by the formation of four plastic hinges at the nodes of the frame. 

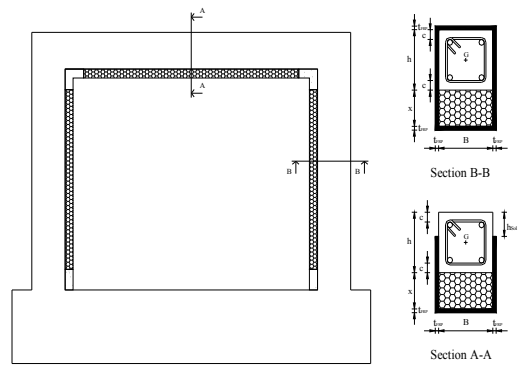

Figure 4: Reference r.c. frame specimen.

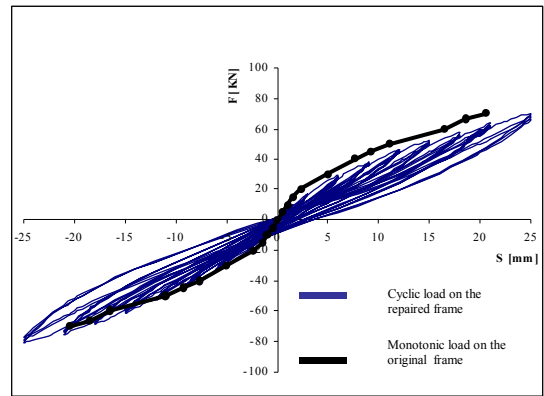

Figure 5: Monotonic test on the original frame.

\subsection{Strengthened sample}

To enhance the bearing capacity of both the columns and the beam, the CFRP was centrifuged by inserting a polyurethane element $6 \mathrm{~cm}$ thick, glued to the internal face of the mesh of the original r.c. frame (Fig. 6). The polyurethane (PUE element), commonly used in civil engineering as thermal or acoustic insulating material, presents poor mass also for great thickness and permit us to maintain the mass of the whole system unchanged. To insert this element each structural element was, previously, cleaned and smoothed, by means of a layer of an epoxy-based primer, like MapeWrap 31. Although the polyurethane seems to be suitable for our scopes, in reality it is a very light, porous plastic material and fails for very low loads; hence the CFRP seal is not guaranteed. For this reason, some anchorage blocks were placed at the extremity of each structural element. The blocks were constituted by reoplastic mortar connected to the original beams by means of four steel plugs, for each side, $120 \mathrm{~mm}$ long, and $70 \mathrm{~mm}$ width, capable of absorbing the slip generated by the shear strength at the bonding interface between CFRP and main structure.

The carbon fiber sheets were then glued to the structural element, beam or column by following the order below:

A layer of Uniaxial CFRP sheet (like Mapei 40/300) was glued at the intrados of the frame beam and bonded around the borders. The choice of the uniaxial sheet is due to its capacity to give a good flexural resistance along the tension face.

A second layer, placed above the previous one, is constituted by Biaxial $20 / 230$ sheet and it was glued on the lateral face of the beam. It gives resistance in two directions and absorb all the shear strength of the structural member.

A layer of Uniaxial CFRP 40/300 was glued at the intrados of the columns on the polyurethane in order to improve their flexural resistance while the second layer, constituted by Biaxial 20/230 sheet, was glued all around the columns as wrapping

Finally, a Quadriax 30/760 sheet is glued at the nodes' frame in order to provide a U-anchorage system to give an effective solution for the case in which 
the bonded length of FRP composites is not sufficient to develop its full capacity.

Figures 7-9 display some phase during the strengthened specimen's preparation. The tests consisted of evaluating the resistance capacity of the strengthened r.c. frame. To this aim a constant vertical load of $100 \mathrm{kN}$ was applied on each column while the horizontal load was applied gradually by means of a hydraulic jack of $250 \mathrm{kN}$ of capacity, capable of imposing on the main structure, controlled displacements of $+/-125 \mathrm{~mm}$ by sinusoidal, quadratic or triangular waves. Once the frame in elastic domain was tested the load was incremented up to the plastic phase. The first cracks are visible at the column base located on the right side, for a load of $60 \mathrm{kN}$.
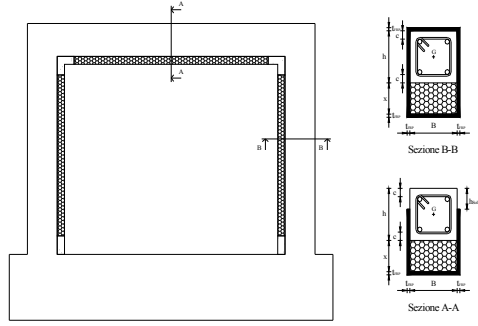

Figure 6: Scheme of the mesh frame with reinforced members.

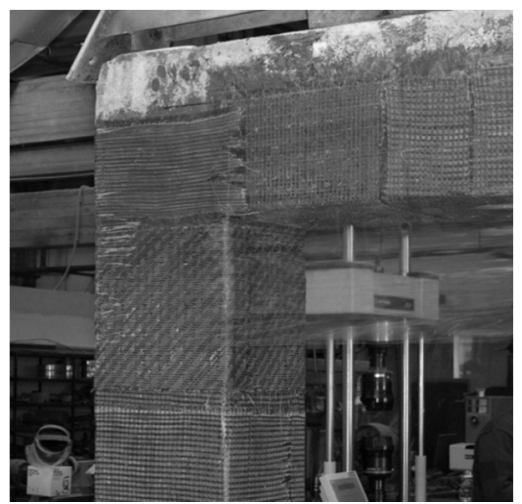

Figure 8: View of the strengthened Figure 9: The system instrumented for beam-column node.

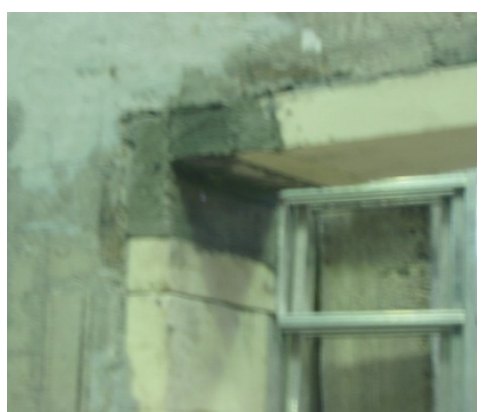

Figure 7: Reoplastic block at the frame nodes.

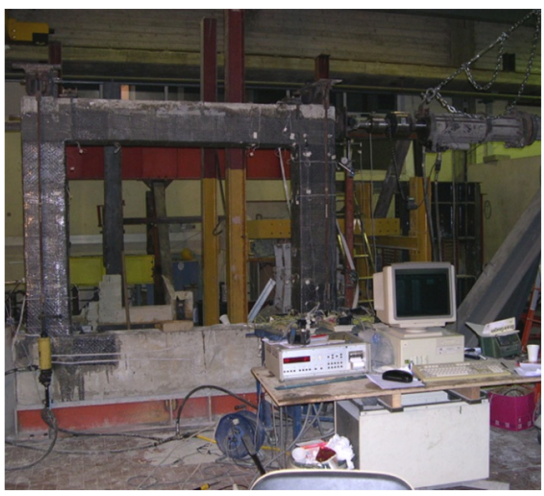
the test.

When the load is incremented, the crack dimensions grow up to the plastic hinge formation but this phenomena was anticipated by the de-bonding that occurred between CFRP and its support. The second plastic hinge occurred on the column located on the left side, for a load equal to $70 \mathrm{kN}$. More cracks are visible for further increment of load all along the frame but it can still bear a load of more than $80 \mathrm{kN}$. 
The test was stopped at a load equal to $92 \mathrm{kN}$ for a maximum displacement of $19.50 \mathrm{~mm}$ correspondent to the ultimate displacement achieved in the test carried out on the non-strengthened model. But from numerical study carried out on the frame was obtained a maximum load much higher and up to $12 \mathrm{kn}$ and a related displacement equal to $35 \mathrm{~mm}$. Figure 10 represents the horizontal load displacement curve obtained from the test and compared to the original frame behavior.

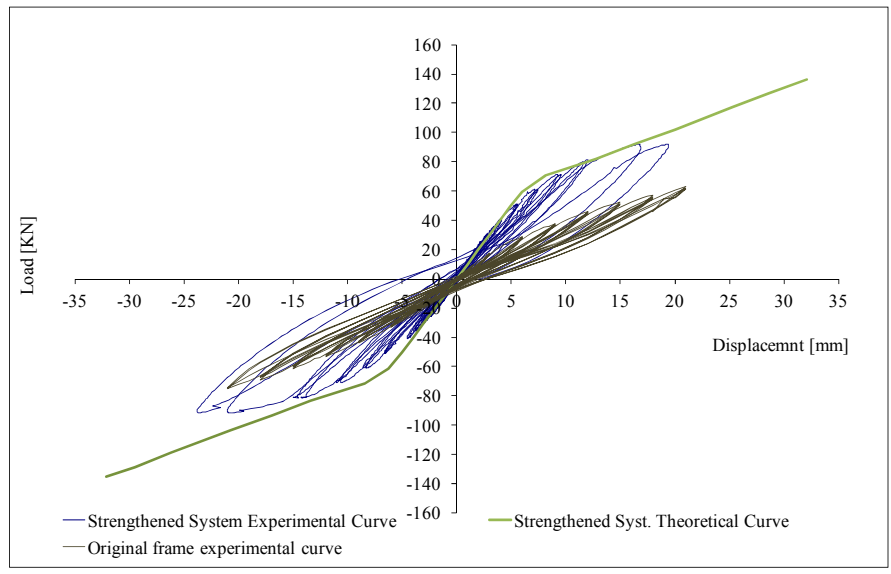

Figure 10: Comparison between reference and strengthened frame by C-FRP.

\section{Application to the real structures}

The retrofitting project design regards a two storey building realized with frame only in one direction. A plan view of the building is reported in figure 11.

The villa, which was used for residential purposes, consists of $\mathrm{n} .2$ floors above ground the first of which is elevated above the ground level and it is home to a total of no. 2 single-family housing.

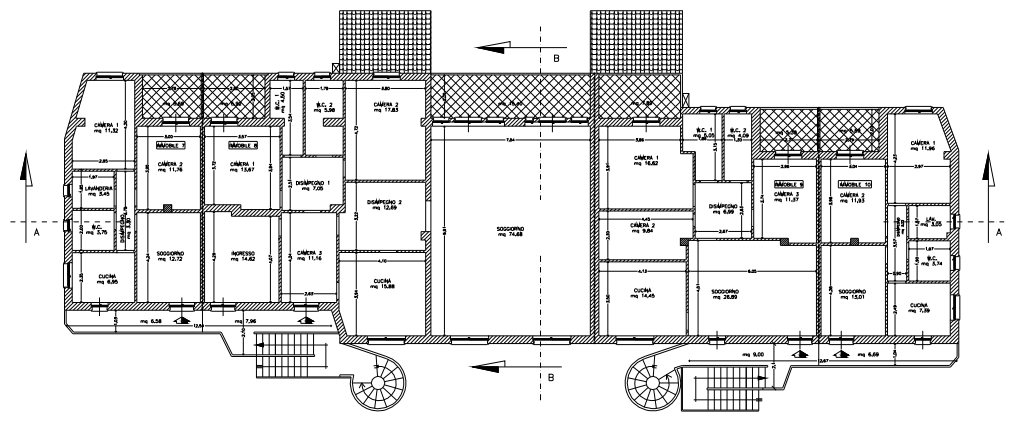

Figure 11: Plan of existing building to be strengthened against seismic hazard. 
The foundation structure is in continuous beams of $30 \mathrm{~cm}$ thick and $70 \mathrm{~cm}$ depth.

In light of the findings of the preliminary experimental investigations and subsequent additional investigations, carried out on elevated structures on the ground and seabed, and on the basis of the calculation of the structure carried out only under vertical loads only, it has been possible to calibrate the retrofitting and strengthening project.

The deficient transversal connection among the longitudinal frames (fig. 12) were overcome by acting directly on the slab floor. It is expected to merge multiple slab beams ( 2 or 3 according to need) with the use of a lightweight material such as PUE element, or as the same pot, and then applying the carbon fiber sheets.

Figure 12 also highlights, in red, the structural elements not capable of sustain the solicitations produced by seismic input. Besides, the cross sections of the existing frame was increased and strengthened by means of the proposed technology. A series of alternative interventions were also required to the aim of an efficient retrofitting. Figure 13 illustrates the plan of the structural interventions provided on the building.

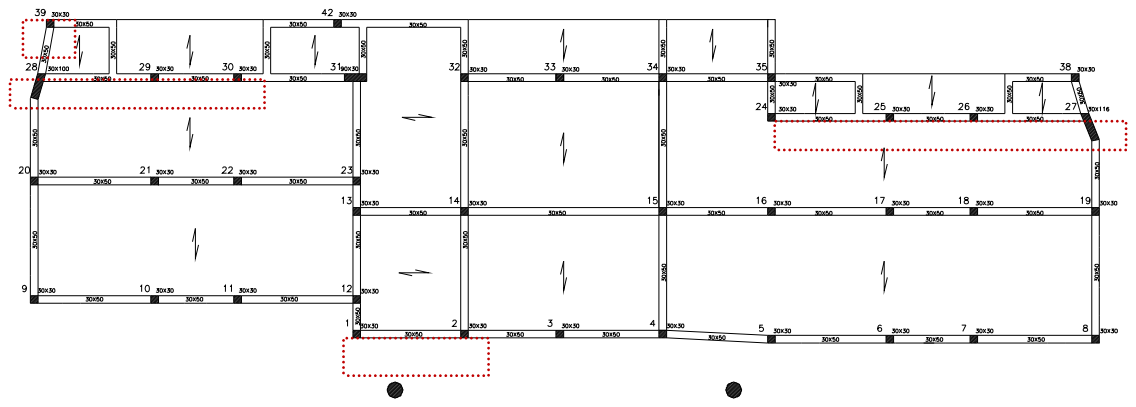

Figure 12: Structural elements in the building. Poor connection along transversal direction.

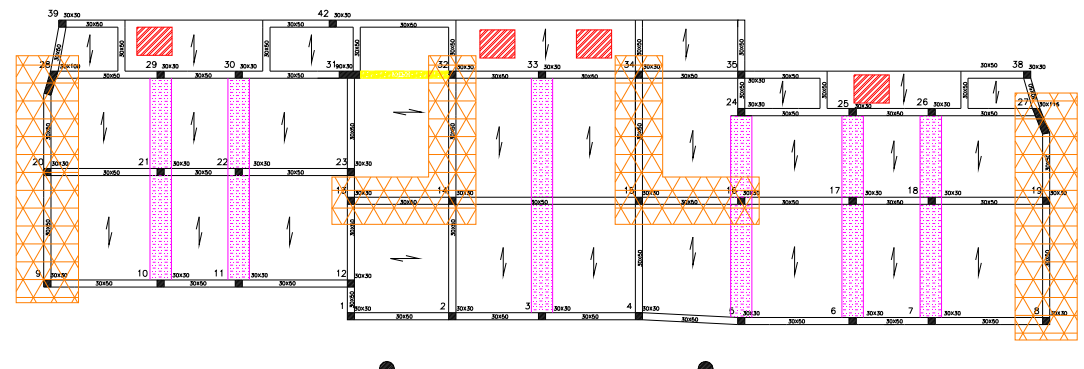

Figure 13: Plan of interventions - orange: strengthening of existing frames by means of the proposed technology; pink: slab beams merging by CFRP. 
Namely, from the structural point of view, in order to improve the seismic behaviour of the building, the following solutions were adopted:

- Transversal connections among the longitudinal frames, by means of carbon fiber strips directly bonded at the intrados of the slab beam, to ensure a global collaboration of the reinforced concrete structure (Fig. 14).

- Strengthening of fiber-reinforced frames with the insertion of PUE element then wrapped by CFRP, placed at strategic points in the structure according to the numerical relevancies, to ensure a wider dissipative structural capacity (Fig. 15).

- Structural reinforcement in C.F.R.P. for those beams that fail due to lack of adequate reinforcement, to withstand the static and dynamic loads.

In this way, a reduced load transferred from the slab to the pillars is also obtained.

\section{Existing slab}

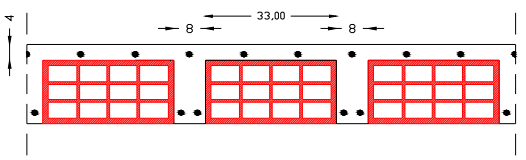

Elimination pots and deteriorated concrete cover

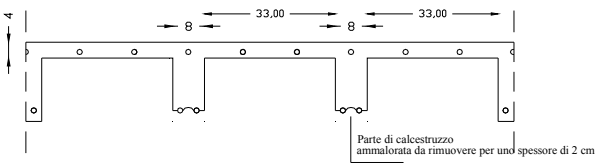

Treatment of steel reinforcement makeover concrete cover and insert of PUE

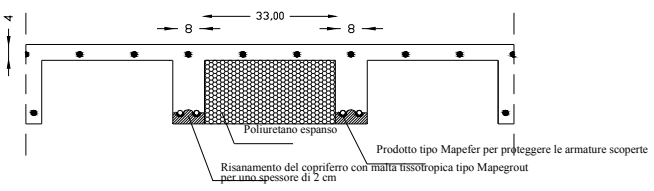

Inserting of uniaxial carbon fibers

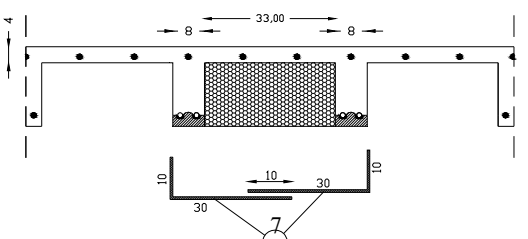

Strenghtened slab

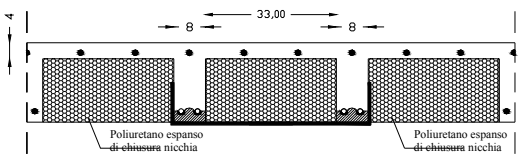

Figure 14: Merging of slab beams. 


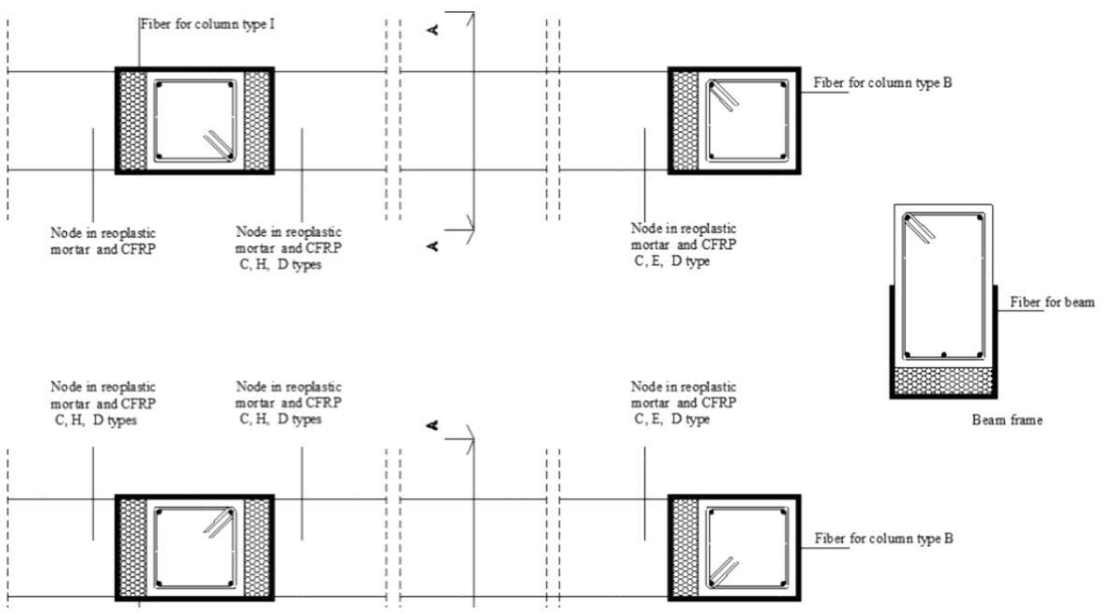

Figure 15: Strengthening frame by PUE and CFRP.

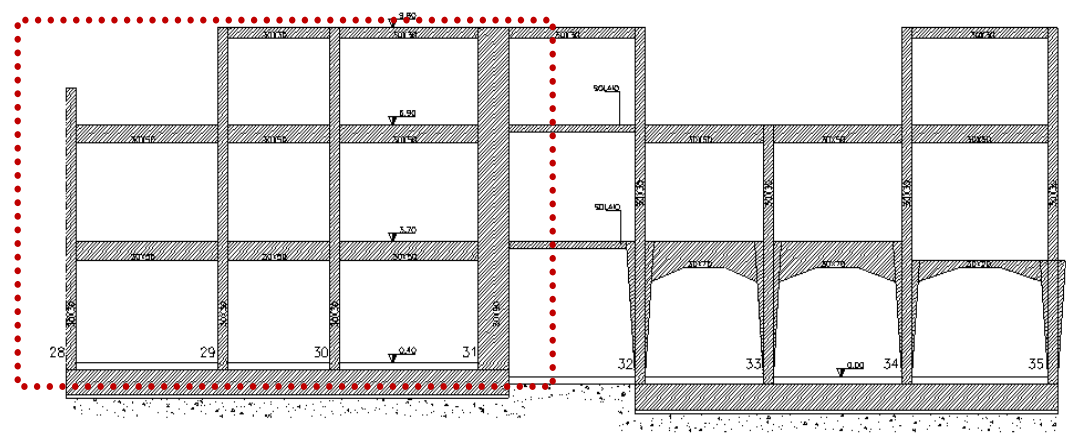

Figure 16: Structural section view of the building.

Frames 28-30 represented in Figure 16 show columns with cross section of $30 \times 30 \mathrm{~cm}$ and beams $30 \times 50$. Those elements are not able to face the solicitations derived by the acting loads. But, by applying the proposed procedure (Figs 1720) we can observe that the solicitations are all inside the M-N related to the reinforced section just with one or two plies of CFRP sheets both in the case of the columns (Fig. 21) and of the beams (Fig. 22). 


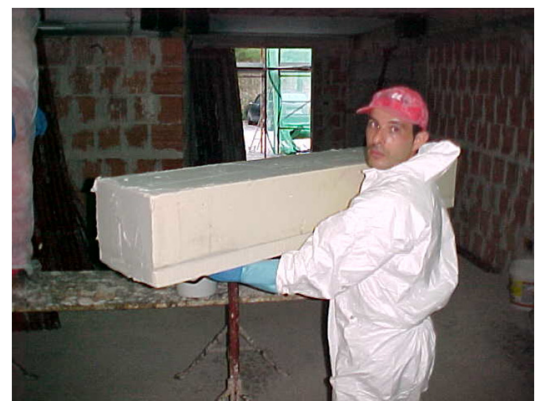

Figure 17: PUE element: light weight.

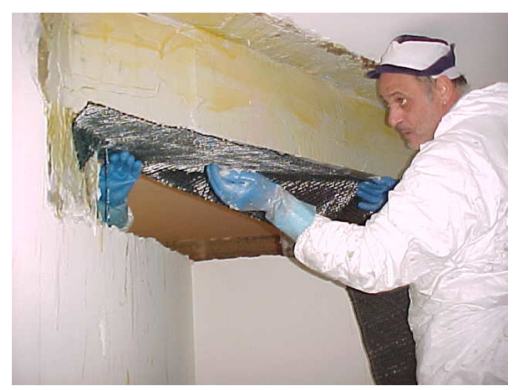

Figure 19: Application of uniaxial CFRP layer.

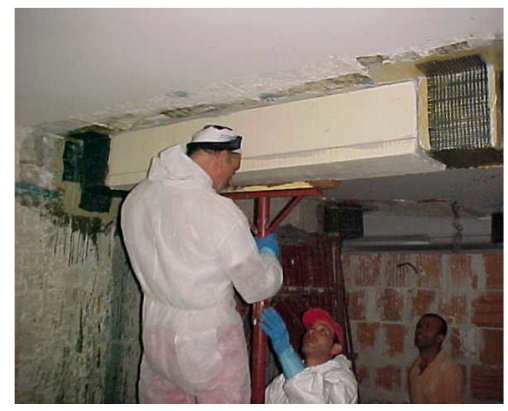

Figure 18: Application of PUE element.

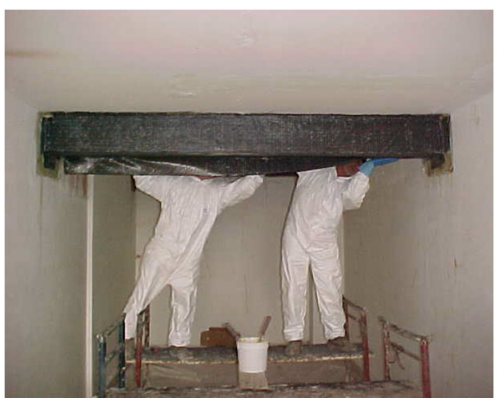

Figure 20: Beam after strengthening procedure.

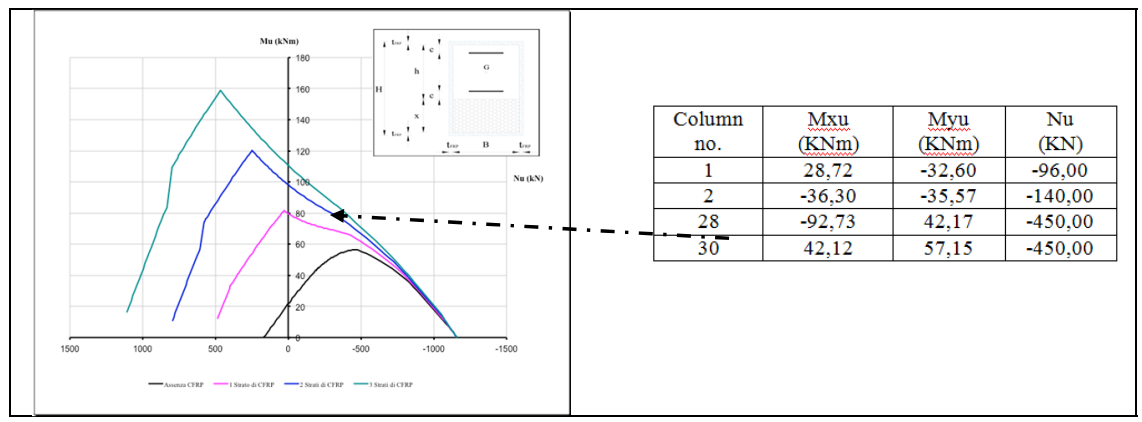

Figure 21: $\quad$ M-N domain for columns - solicitations in the columns. 


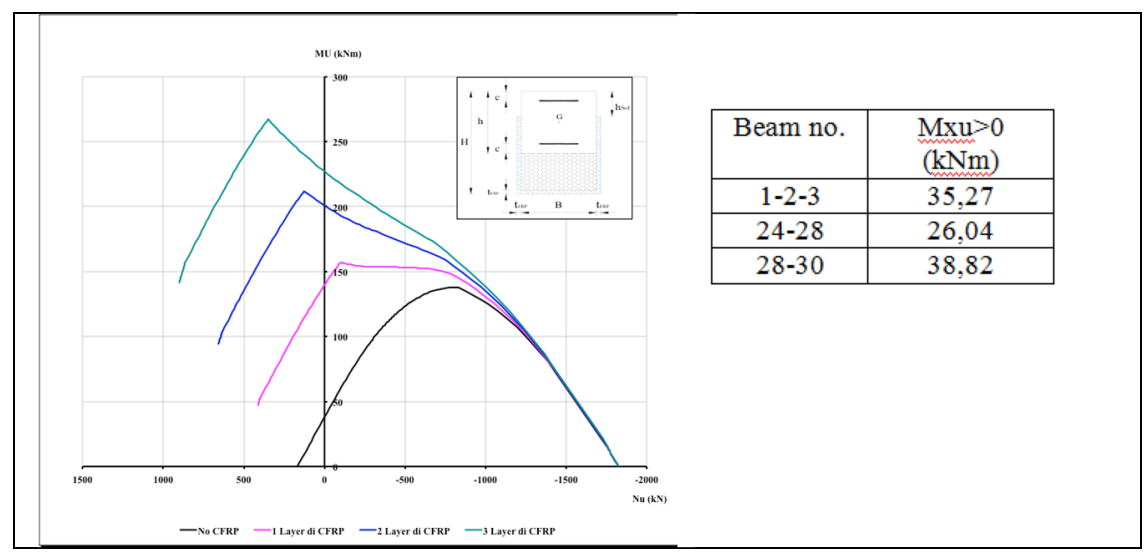

Figure 22: $\mathrm{M}-\mathrm{N}$ domain for beams - solicitations in the beams.

\section{Conclusions}

The paper refers to a new technology to be employed in r.c. frame structures. We can say that the proposed technology seems to be very efficient both in terms of flexural enhancement and of bearing capacity as well as in terms of ductility due to columns confinement. We can get an increase in the bending moment, shown in the previous chapters -up to three times greater than the original frame- and the compression strength. The test, carried on the strengthened frame, gives good results in terms of stiffness as well as in terms of plastic dissipation. In fact, even when plastic hinges have occurred at the frame nodes, the structure is still able to bear further strengths and loads in respect to the original frame. The proposed technology displayed an increasing of the stiffness, flexural and shear resistance (El Maaddawy and Sherif [6], Sundarraja and Rajamohan [7]) as well as a formidable contribution for addressing the tensile stresses on the columns. In conclusion, the study treated above and the experimental results permitted us to demonstrate the great potential of this kind of reinforcement for r.c. lacking structures and encourages its use, especially in the case of seismic retrofitting of the existing framed structures under in plane seismic loads as demonstrated in this paper.

The proposed technology was, in fact, employed in a real structure. Its validity was proved by acceptance tests carried out on the structure by dynamic input.

\section{References}

[1] Galicia H.I., Jara J.M., Negro P. Strengthening of a four-story building model using C-FRP fabrics. In: Proc. of the 8th international symposium on fiber-reinforced polymer reinforcement for concrete structures. 2007. 
[2] Pendhari S.S., Kant T., Desai Y.M. Application of polymer composites in civil construction: a general review. Comput Struct 2008; 84(2): 114-124, 2008.

[3] Della Corte G., Barecchia E., Mazzolani F.M. "Seismic upgrading of RC buildings by FRP: full-scale tests of a real structure”. J Mater Civ Eng 2006; 18(5): 659-669, 2006.

[4] Anania L., Badala' A., Failla G. "Increasing of the Flexural Performance of RC Beams strengthened with C-FRP Materials" Construction and Building Materials, 19: 55-61, 2005.

[5] Anania L., D’Agata G. "Numerical investigation on a new CFRP technology for the upgrading of the RC framed structures" APCBEE Procedia (2013) ICCEN, 2014.

[6] El Maaddawy, T., Sherif, S. "FRP composites for shear strengthening of reinforced concrete deep beams with openings", Composite Structures, vol. 89, no. 1, pp. 60-69, 2009.

[7] Sundarraja, M.C., Rajamohan, S. "Strengthening of RC beams in shear using GFRP inclined strips - An experimental study", Construction and Building Materials, vol. 23, no. 2, pp. 856-864, 2009.

[8] Jeong S.H., Elnashai A.S. Numerical assessment of an irregular RC full scale 3D test structure. Mid-America Earthquake Center, Dept. of Civil and Environmental Engineering, University of Illinois at Urbana-Champaign, Urbana (Illinois, USA): p. 146, 2004. 